\title{
CALIDAD DE LA METODOLOGÍA DIDÁCTICA POR MEDIO DE ENTORNOS VIRTUALES DE APRENDIZAJE EN LA FORMACIÓN DE AGENTES EDUCATIVOS
}

\author{
Sonia $M^{a}$ Santoveña Casal
}

UNED

\begin{abstract}
RESUMEN: Se presentan los resultados obtenidos al analizar la calidad de la metodología didáctica en entornos virtuales de aprendizaje de cinco asignaturas de la Licenciatura de Pedagogía y de la Diplomatura de Magisterio de las especialidades de Infantil y Primaria. La muestra está formada por 5 cursos virtuales y 153 usuarios. El instrumento de recogida de información ha sido un cuestionario y se ha realizado un análisis descriptivo y de varianza. Los contenidos, el uso de las herramientas de comunicación y la calidad de las guías docentes, son aspectos muy valorados por los usuarios.
\end{abstract}

PALABRAS CLAVE: Metodología didáctica, entornos virtuales de aprendizaje, formación, educación.

\section{QUALITY OF DIDACTIC METHODOLOGY THROUGH OF VIRTUAL LEARNING ENVIRONMENTS IN THE EDUCATION AGENT TRAINING}

\begin{abstract}
The results obtained by analyzing the quality of teaching methodology in virtual learning environments of five subjects for the Pedagogy Degree and Pre-Primary and Primary Teaching Education Bachelor Degrees are introduced. The sample consists of five online courses and 153 users. The tool used for the data collection has been a questionnaire. A descriptive analysis and a variance analysis have been carried out. However, contents, the use of communication tools and the quality of the teaching guides are very appreciated by users of the courses.
\end{abstract}

KEYWORDS: Didactic methodology, virtual learning environments, training, education. 


\section{INTRODUCCIÓN}

La integración de las Tecnologías de la Información y la Comunicación (TIC) en el sistema educativo de la mayoría de las universidades ha potenciado el desarrollo e implantación de planes formativos a distancia y, por tanto, la producción de cursos virtuales como apoyo a la docencia presencial. En este contexto, son varias las investigaciones realizadas con el objetivo de conocer cuáles son las características que debe presentar un curso virtual para considerarse una herramienta de aprendizaje de calidad.

Como indican Ellis, Jarkey, Mahony, Peat y Sheely (2007) es necesario preguntarse ¿Cómo el eLearning ayuda a los estudiantes a comprender mejor el material didáctico que está estudiando? ¿Qué estrategias se están adoptando con los estudiantes que tienen éxito en el proceso de enseñanza-aprendizaje en red? ¿Qué estrategias son necesarias adoptar? Los autores afirman que es importante estudiar cómo el curso virtual Ilega a ser un complemento del aprendizaje presencial. Por otra parte, consideran necesario analizar la relación existente entre las actividades de apoyo y los materiales y conocer si los contenidos son elementos de refuerzo del aprendizaje.

\subsection{Variables influyentes en el proceso de enseñanza-aprendizaje en red}

En este marco, se han desarrollado distintas iniciativas de evaluación de la calidad de los cursos virtuales, identificando algunas variables consideradas influyentes en el proceso de enseñanza-aprendizaje en red, como son: los contenidos didácticos, estilos de aprendizaje, el aprendizaje constructivista y significativo, la variedad y riqueza de las actividades y del sistema de evaluación, el proceso de interacción y comunicación y la motivación.

Recientes investigaciones han destacado la importancia de presentar contenidos de calidad en los cursos virtuales (Crissman, 2002; DeLotell, Millam, Reinhardt, 2010; Dykman y Davis, 2008; Santoveña, 2007) y, como indica Ellis (2010), la relevancia de atender al tipo de contenidos presentados, como puede ser el añadir contenidos complementarios, no relacionados de manera directa con los resultados de aprendizaje, pero que apoyen la adquisición de conocimientos por parte del estudiante.

Una de las variables enfatizadas como influyente en el rendimiento de los estudiantes en los cursos virtuales, son los estilos de aprendizaje (Alonso, Gallego y Honey, 2002; Part, Crocker, Nussey, Springate y Hutchings, 2010). Part, Crocker, Nussey, Springate y Hutchings (2010) realizan un estudio con el objetivo de conocer cómo mejorar el aprendizaje de los estudiantes por medio de programas educativos virtuales y concluyen que cuando las estrategias de aprendizaje diseñadas no son coincidentes con las preferencias de aprendizaje de los estudiantes, el rendimiento de los alumnos es menor.

Otras de las variables analizadas como influyentes en el aprendizaje de los estudiantes en red han sido los procesos de comunicación y de interacción. Son números los autores que han subrayado la influencia de estas variables sobre el aprendizaje de los estudiantes en red (Biner, Barone, Welsh y Dean, 1997; Crissman, 2001; DeLotell, Millam y Reinhardt, 2010; Garrison, Anderson y Archer, 2001; Gunawardena, Linder-VanBerschot, LaPointe y Rao, 2010; Johnson, Aragón, Shaik, 
y Palma-Rivas, 1999; Moore, 1989). Las respuestas rápidas del profesor (Biner, Barone, Welsh y Dean, 1997), proporcionar información sobre el progreso de manera adecuada y cuidar la interacción entre alumno-profesor (Johnson, Aragón, Shaik, y Palma-Rivas, 1999), son algunos de los aspectos que facilitan el aprendizaje.

Además, en el estudio de la calidad de los entornos virtuales de aprendizaje, se han estudiado las actividades didácticas y el proceso de evaluación presentado en el curso. Knight (2007) afirma que la incorporación de más actividades, con el objetivo de evaluar el rendimiento de los estudiantes en el curso en línea, influye positivamente sobre el rendimiento obtenido.

El estudio de la relación entre la motivación del estudiante y el rendimiento en el aula virtual ha sido reiteradamente presentado en las principales investigaciones. Los resultados indican que la motivación, inteligencia, esperanza, optimismo y la fijación de metas están influyendo en el rendimiento de los estudiantes en red (Bressler, Bressler y Bressler, 2010) y que el nivel de satisfacción del alumnado es una variable determinante en la mejora del rendimiento de los estudiantes online (Biner, Barone, Welsh y Dean, 1997; Gunawardena, Linder-VanBerschot, LaPointe y Rao, 2010; Knight, 2007; Puzziferro, 2008).

Es interesante destacar la revisión que realizan Bressler, Bressler y Bressler (2010) sobre la literatura disponible relativa a las variables influyentes en el éxito de los cursos virtuales: las actitudes de los estudiantes (Hogan, 1997; Hoy, Tarter y Hoy, 2006); el optimismo, la esperanza y el apoyo social (Juntunen y Wettersten, 2006; RogersonRevell, 2007; Westburg y Martin, 2003); los objetivos (Harackiewicz, Barron, Tauer, Carter y Elliot, 2000; Pekrun, Elliot y Maier, 2006); el establecimiento de metas, el optimismo, la esperanza y el optimismo académico (Hogan, 1997; Smith y Hoy, 2007).

La motivación determina el interés hacia el estudio, favoreciendo la participación del alumno en el proceso de enseñanza-aprendizaje de forma activa y continua y, por tanto, posibilitando la adquisición del conocimiento. La motivación intrínseca (Atherton, 2010), el interés, el compromiso del estudiante en el proceso de aprendizaje (Rosi, 2000), poner en marcha estrategias que faciliten la reflexión, la solución de problemas, la toma de decisiones (Ngeow y Kong, 2003), la satisfacción y la compresión (Nelson Laird, Shoup, Kuh y Schwarte, 2008), la comprensión y el establecimiento de relaciones entre los conceptos (Draper, 2009), han sido otras de las variables consideradas determinantes en el proceso de aprendizaje en línea (citado en DeLotell, Millam, Reinhardt, 2010).

DeLotell, Millam, Reinhardt (2010), estudian la influencia de distintas estrategias de aprendizaje profundo en la retención en los estudiantes de un Online Business Courses. Por medio de los foros de discusión, los profesores intentan promover el proceso de aprendizaje profundo que tiene lugar cuando los alumnos conectan con los contenidos del curso, los valoran y son capaces de llevarlos a la práctica. Los autores afirman que cuando los estudiantes realizan un aprendizaje profundo de los contenidos mejoran su tasa de retención. Entre las estrategias de instrucción que destacan como más efectivas destacan la reflexión y la motivación.

Otros autores que apoyan la importancia del aprendizaje profundo son, por un lado, Crissman (2001) que concluye que a pesar de la importancia del plan de estudios y de los contenidos en el logro de un aprendizaje profundo, es más relevante 
la colaboración entre estudiantes y profesores y, por otro, Garrison, Anderson y Archer (2001) que afirman que existe una unión evidente entre el pensamiento crítico, la interacción social y el aprendizaje profundo.

En el marco del constructivismo se concibe un proceso de aprendizaje donde el responsable último es el estudiante. Se considera que la educación tendrá como principal función facilitar el acceso al conocimiento y que la construcción del conocimiento será el resultado de un proceso compartido entre docente y estudiante, donde, bajo la guía y orientación del profesor, será la actividad constructiva del alumno la que promueva un aprendizaje eficaz (Coll, Martín, Mauri, Miras, Onrubia, Solé, et al., 1999).

Knight (2007) concluye, en un estudio realizado en educación de postgrado en The Open University, que la tutorización virtual no influye (ni positiva, ni negativamente) en el rendimiento, retención o éxito de los cursos formativos y que, por tanto, los principios y variables tradicionalmente considerados influyentes en la retención en los estudiantes siguen siendo válidos, con independencia del medio virtual o presencial utilizado. Por tanto, se puede afirmar que el éxito o fracaso de un método formativo depende no exclusivamente al cambio tecnológico, sino que tenemos que atender a variables más tradicionales.

\subsection{La Universidad de Oviedo}

La Universidad de Oviedo, como el resto de las universidades españolas, está inmersa en el proceso de adaptación al EEES. Es evidente el esfuerzo constante que está realizando por integrar las TIC en su sistema educativo. La universidad ofrece 84 titulaciones de Grado que siguiendo los principios establecidos desde el Espacio Europeo de Educación Superior, se basan en un sistema de enseñanza de grupos reducidos y de contenido eminentemente práctico. Encontramos una oferta en postgrado de 34 másteres oficiales y 3 Erasmus Mundus. Hay que destacar que la universidad oferta 37 doctorados adaptados al EEES y se han defendido en este marco 150 tesis doctorales (Universidad de Oviedo, 2009).

El sistema educativo de la Universidad de Oviedo está basado en una formación universitaria presencial que ofrece a los profesores y alumnos recursos virtuales complementarios. Los cursos virtuales de la Universidad tienen carácter voluntario y, por tanto, son utilizados exclusivamente por los docentes que los solicitan. Consecuentemente, no todas las asignaturas disponen de espacio en red. La plataforma virtual de enseñanza es Uniovi Virtual, originalmente denominado Aulanet, creada en 1999. Los datos indican que actualmente aloja más de 2.000 asignaturas, 1.200 profesores y más de 25.000 estudiantes (Vicerrectorado de Informática y Comunicaciones). Uniovi Virtual, está basada en la plataforma Moodle que como destaca Llorente (2007) ofrece un espacio virtual viable y eficaz. Un ejemplo de integración de Moodle en el sistema educativo, es el uso y la mejora de la plataforma realizado por The Open University, donde se incorporó para impartir docencia a distancia a 180.000 estudiantes. Sclater (2008) afirma que aunque ha sido necesario adaptar y mejorar el entorno para integrarlo con el sistema universitario, la universidad valora que sus modificaciones han derivado en una integración del producto básico para el beneficio de otros y el crecimiento continuo de la comunidad Moodle. 
La Facultad de Formación de Profesorado y Educación, de la Universidad de Oviedo, durante el curso académico 2006/07 diseñó varias iniciativas para comenzar a trabajar en la adaptación de la Licenciatura en Pedagogía al Plan de Bolonia. Se desarrolla el Plan de Mejora del Primer Ciclo de la titulación de Pedagogía, donde los equipos docentes, de los tres primeros cursos, comienzan a trabajar en el curso académico 2007-2008 (Diego, Iglesias y Molina, 2010).

Molina, Iglesias y Diego (2007) presentan un proyecto de innovación, desarrollado durante el curso 2005-2006 en la asignatura Sistemas de Información y Documentación sobre Educación de primero de la Licenciatura de Pedagogía. Después de elaborar los materiales didácticos de la asignatura, los incorporan al aula virtual en la plataforma AulaNet. La comunicación con los estudiantes se desarrolló a través del foro y del correo de la plataforma. Algunos de los resultados obtenidos indican que el $56,3 \%$ de los estudiantes ha consultado el material por lo menos una vez al mes y el $74 \%$ considera que la herramienta favorece el aprendizaje autónomo. Las profesoras concluyen que el material didáctico ha resultado de utilidad para el aprendizaje pero que las clases presenciales es el espacio óptimo para seguir las explicaciones y plantear dudas. Posteriormente, los mismos autores, durante el curso académico 2008-2009 amplían la muestra de estudio. El objetivo del proyecto es el estudio del desarrollo de la competencia gestión de la información, en tres asignaturas de la Licenciatura de Pedagogía. Los resultados indican que el alumnado tiene dificultades para construir su propio conocimiento, además de observar que existe una « (...) tendencia a compartimentar la información obtenida en función de la asignatura así como una propensión a utilizar las palabras de otros autores sin citar su procedencia.»(Diego, Iglesias y Molina, 2010, pp. 5).

En 2009, Álvarez-García, Álvarez Pérez y Rodríguez Pérez, presentan el diseño de un programa de prácticas de la asignatura «Psicología de la Educación y del Desarrollo en la edad escolar», de la Diplomatura de Magisterio, especialidad de Educación Musical para el aula virtual del curso académico 2008-2009. Encuentran que los estudiantes valoran positivamente la posibilidad de realizar las prácticas de la asignatura virtualmente, puesto que les facilita la integración de los estudios en su vida cotidiana. Por otra parte, se resalta la función del foro como medio de comunicación para publicar novedades de interés para el grupo y el planteamiento de consultas que posteriormente, en muchos casos, son explicadas en las sesiones presenciales.

Partimos de que es necesario conocer la influencia en el proceso de enseñanza, por medio de entornos virtuales de aprendizaje, de variables tales como: contenidos, estilos de aprendizaje, metodología didáctica, actividades, sistema de evaluación, proceso de comunicación e interacción, motivación e interés y el aprendizaje profundo y constructivista. Se puede afirmar que el estudio de la calidad de la metodología didáctica de los cursos virtuales impartidos como complemento a la enseñanza presencial es una necesidad que está promoviendo diferentes estudios e investigaciones con el objetivo de conocer la eficacia de los cursos virtuales como complemento a la enseñanza presencial. Sobre la base de este objetivo se ha desarrollado la investigación presentada en este artículo. En definitiva, el objetivo principal de esta investigación ha sido estudiar la calidad de la metodología didáctica de los cursos virtuales de la Licenciatura de Pedagogía y de las Diplomaturas de Magisterio, especialidad de Infantil y especialidad Primaria, distinguiendo como objetivos específicos los siguientes: 
1. Comparar la calidad de la metodología didáctica de los cursos virtuales dirigidos a la formación de agentes educativos en distintas asignaturas de la Licenciatura de Pedagogía y de la Diplomatura de Magisterio (Infantil y Primaria).

2. Analizar la atención que se realiza en los cursos a los estilos de aprendizaje, el proceso de comunicación e interacción, la riqueza de actividades y el sistema de evaluación.

3. Conocer la calidad de los contenidos, guías docentes y materiales didácticos presentados en los cursos virtuales.

4. Examinar la calidad de la metodología didáctica de los cursos, como recursos que refuerzan la motivación y el aprendizaje constructivista y profundo.

\section{MATERIAL y métOdOS}

\subsection{Análisis estadístico}

Se lleva a cabo un análisis descriptivo de las principales variables de clasificación (Sexo, Pedagogía/Magisterio, Curso que estudia/instruye, Asignatura, Usuario, Años de experiencia con las Nuevas Tecnologías) y de las respuestas a los ítem del cuestionario.

En segundo lugar, se desarrolló, un análisis de varianza con el objetivo de conocer la relación existente entre la variable de Calidad de la Metodología didáctica, medida a través del cuestionario, con las variables: Sexo, Usuario, Asignatura, Pedagogía/Magisterio, Años de experiencia. Además, la Calidad de la Metodología didáctica se ha relacionado con dos factores fijos: Años de experiencia y Asignatura y, Usuario y Asignatura. Para finalizar, se han utilizado tres tipos de pruebas de comparación de medias: T de Student; ANOVA de un factor y, ANOVA de dos factores.

\subsection{Instrumentos de recogida de información: Cuestionario}

El cuestionario es el resultado de la modificación de un cuestionario previo utilizado en la evaluación de los cursos virtuales de la UNED, que posteriormente fue adaptado a las características del sistema educativo de la Universidad de Oviedo, siguiendo las indicaciones de los expertos consultados. La calidad de las aulas virtuales son evaluadas por medio de tres aspectos principales: calidad general del entorno virtual (WebCT), de la Metodología didáctica y la calidad Técnica. Ante la imposibilidad de sintetizar todos los resultados obtenidos en la investigación, en este artículo se presenta exclusivamente los resultados de la evaluación de la Calidad de la Metodología didáctica. La herramienta está formada por 46 preguntas cerradas y 8 preguntas abiertas. Las preguntas cerradas tienen 5 opciones de respuesta que indican la frecuencia o intensidad con la que el curso cumple esa característica: 1 (Nada); 2 (Muy poco); 3 (Algo), 4 (Bastante) y, 5 (Mucho). Las 5 preguntas abiertas pretenden enriquecer la información relativa a la utilidad y satisfacción de los recursos digitales, además de recoger sugerencias de mejora de los cursos.

El estudio de la Calidad de la Metodología didáctica, objetivo de este artículo, está constituido por 12 preguntas cerradas que abarcan aspectos tales como: contenidos publicados en los cursos, actividades diseñadas, metodología didáctica utili- 
zada, el uso realizado de las herramientas de comunicación (foro, correo, etc.), de las herramientas de estudio y de evaluación, entre otros aspectos (Tabla 1).

Tabla 1. Calidad de la metodología didáctica

El curso virtual ofrece la guía docente actualizada: introducción, equipo docente, objetivos, orientaciones didácticas, actividades, etc.

El curso dispone de un desarrollo de contenidos exhaustivo (introducción, objetivos, esquemas, desarrollo de los temas, actividades, resumen, etc.).

Exactitud y claridad de los contenidos: Los contenidos didácticos son precisos, fiables y objetivos, además de presentarse de forma comprensible.

La metodología didáctica atiende a los distintos estilos de aprendizaje.

Los contenidos y la metodología didáctica utilizada facilitan la transferencia de la información al conocimiento.

Las actividades son variadas, ricas y trascienden el uso de la memoria.

Las actividades facilitan la comprensión y el razonamiento, convirtiendo los contenidos en algo activo y eficiente.

Se utilizan las herramientas de comunicación (foro, correo, etc.) con calidad didáctica que facilitan la interacción y la comunicación entre el grupo de usuarios del curso. Se responde a las consultas realizadas y se da información de interés.

Se utilizan herramientas con calidad didáctica que facilitan el estudio: publicación digital de las actividades a realizar en el curso, organización de grupos de trabajo, creación de foros, se utiliza la herramienta para dar consejos o publicar novedades, etcétera.

Se utilizan herramientas que facilitan la evaluación con calidad didáctica: se da la posibilidad de enviar actividades por red, se envía retroalimentación al alumno de forma precisa y clara, se incluyen preguntas de autoevaluación y/o se ofrecen exámenes interactivos de prueba y se publican.

La metodología didáctica utilizada en el curso potencia actitudes positivas hacia el estudio, mantiene el interés en el seguimiento del curso. El curso motiva al alumno.

La metodología didáctica utilizada en el curso fomenta un aprendizaje activador y constructivo, permitiendo al alumno reconstruir el aprendizaje integrando los nuevos conocimientos con los que ya posee.

El cuestionario puede consultarse en: https://spreadsheets.google.com/viewform?hl $=e n \& f o r m k e y=d H c 0 T W N P M 09 Y R U 5 T T n J x S U Z k U z I N Q k E 6 M A \# g i d=0$.

- Fiabilidad: Se lleva a cabo la aplicación piloto del cuestionario con 40 ítems. Esta versión preliminar del cuestionario fue administrada a una muestra de 20 alumnos de Magisterio de la Universidad de Oviedo. Se realiza un análisis de 
la fiabilidad del cuestionario a través del Alfa de Cronbach para ver la variabilidad de los ítems, obteniendo un Alfa de Cronbach de .825. La consistencia interna de los tres aspectos estudiados ha sido la siguiente: calidad del entorno general: .844; calidad de la metodología didáctica: 907 y calidad técnica de los cursos: .939 .

- Validez: La validación del contenido se desarrolla a través de un grupo de expertos, aplicando una breve entrevista a dos profesores de Pedagogía y Magisterio de la Universidad de Oviedo expertos en nuevas tecnologías. Los criterios de selección establecidos son: competencia, experiencia laboral y formación académica. Se considera que el cuestionario evalúa la utilidad, satisfacción y la calidad didáctica, técnica y la calidad general del entorno de los cursos de forma adecuada. Se realizan varias modificaciones sugeridas por los expertos:

- 5 de las preguntas se consideran excesivamente largas y se sugiere dividirlas en dos.

- Se añaden 3 preguntas relacionadas con la satisfacción con los recursos virtuales.

- Se modifica el formato de presentación.

\subsection{Muestra}

La población está constituida por los profesores, estudiantes y cursos virtuales, publicados en el campus virtual, de Pedagogía y Magisterio, especialidad de Infantil y de Primaria, de la Facultad de Formación del Profesorado y Educación, de la Universidad de Oviedo. La muestra está formada por 8 asignaturas, 3 de Pedagogía, 3 de Magisterio Infantil y 2 de Magisterio de Primaria, publicados en Uniovi Virtual y por 153 usuarios de los cursos: 4 Profesores y 149 alumnos.

Es conveniente tener presente que 3 de las asignaturas comparten espacio virtual, es decir son espacios comunes en los diferentes estudios, en definitiva se evaluarán 5 cursos virtuales. A continuación se presenta una breve descripción:

- Curso virtual A. Se imparte en Magisterio (Primaria e Infantil).

- Curso virtual B. Se imparte en Pedagogía.

- Curso virtual C. Se imparte en Magisterio (Primaria e Infantil).

- Curso virtual D. Se imparte en Pedagogía.

- Curso virtual E. Se imparte en Pedagogía y Magisterio (Infantil).

El estudio descriptivo muestra que el mayor porcentaje de participación en la investigación corresponde al curso virtual A con un 70,6\% (108 de frecuencia) y el menor se observa en las asignaturas $D$ y $E$, con un porcentaje de 5,3\% de las respuestas emitidas. Con un $10 \%$ encontramos el curso virtual $\mathrm{C}$ y con $7,9 \%$ los cursos D y E.

Con relación a la variable Usuario es posible afirmar que hay un mayor porcentaje de participación de alumnos (97,41\%) que de profesores $(2,6 \%), 149$ alumnos en contraste con los 4 profesores. La mayor parte de la muestra es del primer curso $(66,2 \%)$ y de tercer curso $(12,6 \%)$. En tercer lugar, se encuentra el quinto curso $(10 \%)$, segundo curso $(8,6 \%)$ y cuarto $(2,6 \%)$. Con relación a la variable Sexo se 
obtiene que las mujeres, con el $84,4 \%$ (127 de frecuencia), han participado con mayor frecuencia que los hombres (15,9\%, 24 casos).

Con respecto a la participación en función de los estudios realizados, Pedagogía o Magisterio, la mayor parte de la muestra que ha participado es de Magisterio $75,8 \%$ (41,8\% de la especialidad Educación Infantil y 34\% de la especialidad Educación Primaria. El 24,2\% son de la Licenciatura de Pedagogía).

\section{Resultados}

La mayor parte de la muestra afirma tener «más de 6 años» de experiencia con el uso de las TIC (78\%). «Entre 4 y 6 años» de experiencia lo señalan el 18,3\%, «Menos de 2 años» de experiencia lo tienen el 12,4\% de la muestra. En las dos últimas posiciones, con un porcentaje del 9,2\%, encontramos que tienen una experiencia de «Entre 2 y 4 años» y a los sujetos que «No tienen experiencia».

Con relación a los resultados obtenidos en el análisis de la calidad de la metodología didáctica de los cursos, se puede afirmar que todos los ítems que componen el cuestionario muestran una media superior de 3 sobre 5.

Los dos elementos mejor valorados por los usuarios de los cursos en la calidad didáctica están relacionados con la afirmación de que el curso virtual ofrece la guía docente actualizada y con el uso que se realiza de las herramientas de comunicación: El 40,7\% de la muestra está bastante de acuerdo y el 31\% muy de acuerdo con que el curso virtual ofrece la guía docente actualizada (introducción, equipo docente, objetivos, orientaciones didácticas, actividades, etc.) y el 40,6\% de la muestra está bastante de acuerdo y el 31,5\% muy de acuerdo con la afirmación de que «se utilizan las herramientas de comunicación (foro, correo, etc.) con calidad didáctica que facilitan la interacción y la comunicación entre el grupo de usuarios del curso. Se responde a las consultas realizadas y se da información de interés».

El $44,5 \%$ de la muestra está bastante de acuerdo y el $24,7 \%$ muy de acuerdo con la afirmación de que «el usuario puede participar, individualmente y en grupo en las actividades propuestas por los responsables del curso» (D10, media de 3,86).

El $45,5 \%$ de la muestra está bastante de acuerdo y el $16,8 \%$ muy de acuerdo con la afirmación de que «el curso dispone de un desarrollo de contenidos exhaustivo (introducción, objetivos, esquemas, desarrollo de temas, actividades, resumen, etc.)».

El $45,1 \%$ de la muestra está bastante de acuerdo y el 15,4\% muy de acuerdo con que los contenidos son exactos y claros, son «precisos, fiables y objetivos, además de presentarse de forma comprensible»(D13, 3,69 de media).

La media más baja se obtiene en el ítem D16 $(3,50)$ seguido de los ítems: D14 $(3,51)$ y D17 $(3,52)$. Los elementos peor evaluados están relacionados con la afirmación de que las actividades son variadas, ricas y trascienden el uso de la memoria (D16) y con que la metodología didáctica atiende a los distintos estilos de aprendizaje (D14). El 36,6\% de la muestra está sólo bastante de acuerdo y el 31,7\% algo de acuerdo con que «las actividades son variadas, ricas y trascienden el uso de la memoria». El 38,7\% de la muestra está sólo algo de acuerdo y también el 38,7\% está bastante de acuerdo con que «la metodología didáctica atiende a los distintos estilos de aprendizaje». El 33\% de la muestra está sólo algo de acuerdo, el 33\% bas- 
tante de acuerdo y el $14 \%$ muy poco de acuerdo con que «las actividades facilitan la comprensión y el razonamiento, convirtiendo los contenidos en algo activo y eficiente» (D17).

Los datos procedentes del análisis de la variable Sexo, indican que los hombres $(44,29$ de media) valoran mejor los cursos que las mujeres, que obtienen una media de 36,40 , aunque se rechaza la hipótesis de igualdad. El estadístico $t(-2,789)$ y con un nivel crítico bilateral de .008, permite concluir que hay diferencias significativas. Resultados similares aporta la prueba no paramétrica $U$ de Mann-Whitney (.022).

Con relación a la variable Usuario se encuentra que los estudiantes valoran más positivamente los cursos virtuales $(37,81$ de media) que los profesores $(32,50$ de media); sin embargo, estamos ante varianzas iguales. El estadístico $t$ (-.624), con un un nivel crítico bilateral de .533. Se concluye que no hay diferencias significativas. Resultados similares aporta la prueba no paramétrica $U$ de Mann-Whitney (.689).

Los cursos de Magisterio son los mejor valorados (media de 40,95) que los estudios de la Licenciatura de Pedagogía (media de 27,41). El estadístico $t$, toma el valor de -3,524 (nivel crítico bilateral de .001). Se concluye que hay diferencias significativas en las respuestas. Los resultados son apoyados por la prueba $U$ de Mann-Whitney (.002).

Con relación a la variable Asignatura, se concluye que la asignatura A es la que mayor media obtiene $(40,7)$ y en segundo lugar está la asignatura C (39,93 de media) y la asignatura B $(8,9)$ la de menor media. La prueba de Levene muestra que no hay homogeneidad de varianzas (nivel crítico de .000). Los resultados confirman la diferencia de medias con la prueba de Welch (.002) y con Brown-Forsythe (.004). T2 de Tahame corrobora que existen diferencias entre el siguiente pares de medias: A-B (.004) y entre C-B (.008). Con la prueba $H$ de Kruskal-Wallis se encuentran diferencias significativas (sig. asintót.= .001). Concluimos que existen diferencias significativas.

Los elementos mejor valorados del curso virtual A están relacionados con: la calidad de la información presentada en la guía docente (3,79 de media); el uso realizado de las herramientas de comunicación que han facilitado la interacción y la comunicación (3,73 de media); el desarrollo de una metodología que ha reforzado actitudes positivas hacia el estudio y ha conseguido mantener el interés en el seguimiento del curso (3,56 de media) y, además, ha potenciado un aprendizaje activador y constructivo, permitiendo al alumno reconstruir el aprendizaje integrando los nuevos conocimientos con los que ya posee (3,56 de media).

Los resultados en función del Curso que estudia/instruye revelan que los usuarios del $2^{\circ}$ curso son los que más alto evalúan los cursos $(42,15$ de media) y los usuarios de 3 er (media de 28,42) los que bajo los valoran. La prueba de Levene indica que hay diferencias significativas (nivel crítico de .000). La prueba de Welch (.185) y la prueba de Brown-Forsythe (.061) indican que no existen diferencias significativas. Estos resultados son confirmados por T2 Tahame y $H$ de Kruskal-Wallis (sig. asintót.= .206).

Según la variable Años de experiencia con Nuevas Tecnologías, los datos señalan que los que mejor evalúan la metodología didáctica son los que más experiencia tienen con las Nuevas Tecnologías (Más de 6 años) y los que más bajo lo evalúan son los que tienen entre 2 y 4 años de experiencia, aunque no se puede afirmar que existan diferencias significativas entre las medias (valor crítico asociado de .457). 
En el Modelo corregido que se refiere a todos los efectos de los factores $(F$ de .000), nos indica que el modelo explica una parte significativa de la variación observada en la variable Calidad de la Metodología didáctica: el 30,6\% de la variable Calidad de la Metodología didáctica y se puede afirmar que el efecto de interacción entre ambos factores (.005 de valor crítico) es significativo. Además, se encuentran diferencias significativas en las medias de las asignaturas.

En el análisis Usuario y Asignatura, no se encuentra homogeneidad de varianzas (nivel crítico de .000) y el Modelo corregido indica que todos los efectos del modelo tomados juntos explican el $21,7 \%$ de la variabilidad de la variable. El efecto del factor principal Asignatura es significativo.

En ambos análisis factoriales se obtiene que los pares de medias con diferencias significativas según las pruebas de Tukey y Bonferroni son: A-B; A-E; B-C.

\section{Discusıón}

La Universidad de Oviedo ofrece la posibilidad al profesorado de publicar cursos virtuales en Moodle, plataforma evaluada como un entorno flexible que se adapta a las necesidades de los estudiantes. Los cursos virtuales presentan algunas de las características consideradas por los autores, como básicas en el desarrollo e implantación de un proceso de aprendizaje online de calidad: contenidos y el proceso de comunicación e interacción. Aunque, es posible concluir, que es necesario mejorar aspectos como la atención a los estilos de aprendizaje y a la riqueza de las actividades presentadas, con el objetivo de facilitar un mayor rendimiento de los estudiantes en los cursos y de mejorar el sistema de evaluación a través de la variabilidad de las actividades presentadas.

Las investigaciones señalan la importancia de cuidar los contenidos presentados en los cursos virtuales (Crissman, 2002; DeLotell, Millam, Reinhardt, 2010; Dykman y Davis, 2008) y en los cursos analizados se ha encontrado que las guías didácticas ofrecidas son evaluadas como recursos que proporcionan información actualizada (71\% está bastante o muy de acuerdo), además de valorar los contenidos publicados como exhaustivos, exactos, precisos y claros (62,3\% está bastante de acuerdo o muy de acuerdo), además, los materiales son evaluados como objetivos y comprensibles. Estos resultados coinciden con la investigación realizada por Molina, Iglesias y Diego (2007), donde encontraron que el material didáctico ha resultado de utilidad para el aprendizaje y fue evaluado como altamente adecuado y comprensible.

Un segundo elemento destacado por los expertos (Biner, Barone, Welsh y Dean, 1997; Crissman, 2001; DeLotell, Millam y Reinhardt, 2010; Garrison, Anderson y Archer, 2001) en el proceso de aprendizaje en red ha sido el proceso de comunicación e interacción. Los usuarios de los cursos de la Universidad de Oviedo han afirmado (71\% está bastante de acuerdo o muy de acuerdo) que las herramientas de comunicación se utilizan con calidad didáctica, facilitando la interacción y la comunicación y que los profesores responden a las consultas realizadas y proporcionan información de interés.

De aquí se desprende la importancia que tiene publicar materiales didácticos de calidad, contenidos fiables y válidos, en los cursos virtuales y no utilizarlos exclusivamente como medio de comunicación. 
Es interesante destacar que la mayor parte de la muestra considera que a través de los cursos se permite participar en las actividades propuestas, ya sea de forma individual o en grupo. El 69\% de la muestra afirma estar bastante de acuerdo o muy de acuerdo con el curso virtual está facilitando el desarrollo de actividades didácticas. Resultados similares encontraron Molina, Iglesias y Diego (2007), donde el 74\% del alumnado considera que la herramienta AulaNet favorece el aprendizaje autónomo. Podemos concluir que los entornos virtuales, en concreto Moodle, son un medio adecuado para la publicación y distribución de actividades y ejercicios didácticos que utilizados de forma correcta puede facilitarnos el proceso de evaluación continua.

A pesar de encontrar que los usuarios han evaluado positivamente que en los cursos se publiquen actividades y afirmen que se permite participar en estas actividades de manera individual y/o en grupo, se ha visto que uno de los elementos peor evaluados es la consideración de que estas actividades sean ricas, variadas y que trasciendan el uso de la memoria, faciliten la comprensión y el razonamiento, convirtiendo los contenidos en algo activo y eficiente. Además, otro de los elementos con menor valoración ha sido la afirmación de que la metodología didáctica atiende a los distintos estilos de aprendizaje. En definitiva, es necesario mejorar los estilos de aprendizaje, aspecto que como han demostrado algunas investigaciones puede estar influyendo en el rendimiento de los estudiantes (Alonso, Gallego y Honey, 2002; Part, Crocker, Nussey, Springate y Hutchings, 2010). Además, como afirma Knight (2007), será necesario incorporar más actividades con el objetivo de evaluar al alumnado puesto que influye positivamente sobre el rendimiento.

Se han encontrado diferencias significativas entre cursos de Magisterio y los estudios de la Licenciatura de Pedagogía (media de 27,41). Las diferencias se deben a que los cursos mejor valorados A y el $\mathrm{C}$ pertenecen a Magisterio y el peor evaluado ha sido el curso B de Pedagogía.

El curso virtual A presenta calidad en aquellos elementos consideramos importantes por los expertos; es decir, se observa una guía didáctica actualizada y de calidad, se realiza un uso de las herramientas de comunicación que permite la interacción y la comunicación, donde los profesores responden y atienden las consultas realizadas por los estudiantes. Es una asignatura que presta atención a la metodología didáctica, donde se refuerzan actitudes positivas hacia el estudio y ha conseguido mantener el interés en el seguimiento del curso, valorándolo como un curso motivador. Aspecto considerado básico por Bressler, Bressler y Bressler (2010) y Knight (2007) entre otros. Además de potenciar un aprendizaje activador y constructivo, permitiendo al alumno reconstruir el aprendizaje integrando los nuevos conocimientos con los que ya posee.

Las diferencias significativas encontradas entre el curso A y el curso virtual B, implica que el curso B deberá atender y mejorar la mayoría de los aspectos analizados, y sobre todo la metodología didáctica utilizada en el curso como medio para motivar, reforzar el aprendizaje significativo y la atención que se realiza de los estilos de aprendizaje.

Se puede concluir que hay diferentes aspectos que están influyendo en la calidad de los cursos virtuales. La calidad del proceso de comunicación y el uso realizado de 
las herramientas de comunicación, la información general relacionada con la asignatura y/o las guías docentes, la posibilidad de realizar actividades individuales y/o en grupo a través de los cursos y el desarrollo de los contenidos de aprendizaje.

\section{REFERENCIAS BIBLIOGRÁFICAS}

AGUADED, J. I. y CABERO, J. (2002). Educar en red. Internet como recurso didáctico. Málaga: Aljibe.

ALONSO GARCÍA, C.; GALLEGO GIL, D. y HONEY, P. (2002). Los Estilos de Aprendizaje. Procedimientos de diagnóstico y mejora. Bilbao: Mensajero.

ÁlVAREZ-GARCÍA, D.; ÁLVAREZ PÉREZ, L.; NúÑ̃ZZ PÉREZ, J. C.; CEREZO MENÉNDEZ, R.; GONZÁlEZ-CASTRO, P. y RODRÍGUEZ PÉREZ, C. (2009). Diseño y ejecución de un programa de prácticas para la asignatura «Psicología de la Educación y del desarrollo en la edad escolar» a través del campus virtual de la universidad de Oviedo [versión electrónica]. En actas de VII Jornadas de Redes de Investigación en Docencia Universitaria. La calidad del proceso de enseñanzalaprendizaje universitario desde la perspectiva del cambio. Alicante: Universidad de Alicante.

ATHERTON, J. S. (2011). Learning and Teaching. Deep and Surface learning, Extraído el 31 May 2011, de http://www.learningandteaching.info/learning/deepsurf.htm.

BINER, P.; BARONE, N.; WELSH, K. y DEAN, R. (1997). Relative academic performance and its relation to facet and overall satisfaction with interactive telecourses [versión electrónica]. Distance Education, 18 (2), 318-326.

BRESSLER, L. A.; BRESSLER, M. E. y BRESSLER, M. S. (2010). The role and relationship of hope, optimism and goal setting in achieving academic success: a study of students enrolled in online accounting courses [versión electrónica]. Academy of Educational Leadership Journal, 14 (4), 37-15.

CABERO, J. y LÓPEZ, E. (2009). Evaluación de materiales en red en el Espacio Europeo de Educación Superior (EEES). Barcelona: Davinci.

CABERO, J.; MARÍN, V. e INFANTE, A. (2011). Creación de un entorno personal para el aprendizaje: desarrollo de una experiencia. Edutec-e, Revista Electrónica de Tecnología Educativa, 38, 1-13. Extraído el 7 de febrero de 2012, de: http://edutec.re diris.es/Revelec2/Revelec38/creacion_entorno_personal_aprendizaje_desarrollo_ experiencia.html.

CASTELLS, M. (2003). La Galaxia Internet: Reflexiones sobre Internet, Empresa y Sociedad. Barcelona: Debolsillo.

CASTELLS, M. (2008). La Era de la Información. Vol 1. La Sociedad Red (nueva edición). Madrid: Alianza.

COLL, C. y otros (1999). El constructivismo en el aula. Barcelona: Grao.

CRISSMAN, J. (2002). The impact of clustering first year seminars with English composition courses on new students' retention rates [versión electrónica]. Journal of College Student Retention, 3 (2), 137-152.

DELOTELL, P. J.; MILLAM, L. A. y REINHARDT, M. M. (2010). The Use of deep learning strategies in online business courses to impact student retention [versión electrónica]. American Journal of Business Education, 3 (12), 49-55. 
DIEGO, C.; IGLESIAS, M. T. y MOLINA, S. (2010). Una experiencia de gestión de la información histórica educativa en la Universidad de Oviedo. En actas del CiDd: II Congrés Internacional de Didáciques. L'activitat del docent: Intervenció, Innovació, Investigació. Girona: Universitat de Girona.

DRAPER, S. W. (2009). Catalytic assessment: Understanding how MCQs and EVS can Foster deep learning [versión electrónica]. Bristish Journal of Educational Technology, 40 (2), 285-293.

DYKMAN, C. A. y DAVIS. C. K. (2008). Online Education Forum: Part Two - Teaching Online Versus Teaching Conventionally [versión electrónica]. Journal of Information Systems Education, 19 (2), 157-166. Extraído el 31 de mayo de 2011, de http:// proquest.umi.com/pqdlink?RQT $=318 \&$ pmid $=50955 \& T S=1295862466 \&$ clien $\mathrm{tld}=69739 \& \mathrm{VInst}=\mathrm{PROD} \& \mathrm{VName}=\mathrm{PQD} \& \mathrm{VType}=\mathrm{PQD}$.

ELLIS, J. (2010). What Makes e-Learning Tutorials Effective? The Use of Catalytic Content to Support the Learning Process. In J. Sanchez y K. Zhang (Eds.), Proceedings of World Conference on E-Learning in Corporate, Government, Healthcare, and Higher Education 2010 (pp. 80-89). Chesapeake, VA: AACE. Extraído el 31 de mayo de 2011, de http://www.editlib.org/noaccess/35514.

ELLIS, R. A.; JARKEY, N.; MAHONY, M. J.; PEAT, M. y SHEELY, S. (2007). Managing quality improvement of eLearning in a large, campus-based university. Quality Assurance in Education: An International Perspective, 15 (1), 9-23. Extraído el 31 de mayo de 2011, de http://poa1.uned.es/web/ehost/detail?hid=22\&sid=b445e 855-233d-4ac8-a180-b8aa38bf563c\%40sessionmgr12\&vid=1\&bdata $=$ Jmxhbmc9ZXMmc2loZT1laG9zdC1saXZI.

FACULTAD DE FORMACIÓN DEL PROFESORADO Y EDUCACIÓN (2011). Oviedo. Universidad de Oviedo: Información académica. Extraído el 12 de mayo de 2011, de http://pre-c1nn.innova.uniovi.es/formacionProfesorado/infacademica.html.

GARRISON, D. R.; ANDERSEN, T. y ARCHER, W. (2001). Critical thinking, cognitive presence, and computer conferencing in distance education [versión electrónica]. The American Journal of Distance Education, 15 (1), 7-15.

GUNAWARDENA, C. N.; LINDER-VANBERSCHOT, J. A.; LAPOINTE, D. K. y RAO, L. (2010). Predictors of Learner Satisfaction and Transfer of Learning in a Corporate Online Education Program [versión electrónica]. The American Journal of Distance Education, 24 (4), 207-226.

HARACKIEWICZ, J.; BARRON, K.; TAUER, J.; CARTER, S. y ELLIOT, A. (2000). Shortterm and long-term consequences of achievement goals: predicting interest and performance over time [versión electrónica]. Journal of Educational Psychology, 92 (2), 316-330.

HOGAN, R. (1997). Analysis of student success in distance learning courses compared to traditional courses. Annual Conference on Multimedia in Education and Industiy. (Eric Document Reproduction Service No. ED 412 992). Extraído el 31 de mayo de 2011, de http://www.eric.ed.gov/PDFS/ED412992.pdf.

HOY, W. K.; TARTER, C. J. y HOY, A. W. (2006). Academic Optimism of Schools: A Force for Student Achievement [version electrónica]. American Educational Research Journal, 43 (3), 425-446. 
JOHNSON, S. D.; ARAGON, S. R.; SHAIK, N. y PALMA-RIVAS, N. (1999). Comparative analysis of online vs. face-to-face instruction. Proceedings of the World Conference on the $W W W$ and Internet. Chesapeake, VA: AACE.

JUNTUNEN, C. L. y WETTERSTEN, K. B. (2006). Work hope: Development and initial validation of a measure [version electronica]. Journal of Counseling Psychology, 53 (1), 91-106.

KNIGHT, P. (2007). Promoting retention and successful completion on Masters courses in education: A study comparing e-tuition using asynchronous conferencing software with face-to-face tuition. Online Learning, 22 (1), 87-96. Extraído el 31 de mayo de 2011, de http://www.informaworld.com/smpp/section?content= a769989290\&fulltext $=713240928$.

LLORENTE. M. C. (2007). Moodle como entorno virtual de formación al alcance de todos [versión electrónica]. Comunicar, Revista Científica Iberoamericana de Comunicación y Educación, 28, 197-202.

MAJÓ, J. y MARQUÈS, P. (2002). La revolución educativa en la era Internet. Barcelona: Cisspraxis.

MOLINA, S.; IGLESIAS, M. T. y DIEGO, C. (2007). Enseñanza presencial con apoyo de materiales multimedia en la formación de competencias transversales. En actas de las Jornadas de Intercambio de experiencias en docencia universitaria en la Universidad de Oviedo de 2007. Oviedo: Servicio de Publicaciones de la Universidad de Oviedo.

MOORE, G. M. (1989). Three Types of Interaction [versión electrónica]. The American Journal of Distance Education, 3 (2). Extraído el 31 de mayo de 2011, de http://www.ajde.com/Contents/vol3_2.htm\#editorial.

NELSON LAIRD, T. F.; SHOUP, R.; KUH, G. D. y SHWARTZ, M. J. (2008, septiembre). The effects of discipline on deep approaches to student learning and college outcomes. Research in Higher Education, 49 (6), 469-494. Extraído el 31 de mayo de 2011, de http://www.springerlink.com/content/6001612048626503/fulltext.pdf.

NGEOW, K. y KONG, Y. (2003). Learning through discussion: designing tasks for critical inquiry and reflective learning. Extraído el 31 de mayo de 2011, de http:// www.eric.ed.gov/PDFS/ED477611.pdf.

PART, C.; CROCKER, C.; NUSSEY, J.; SPRINGATE, J. y HUTCHINGS, D. (2010). Evaluation of a Teaching Tool - Wiki - in Online Graduate Education. Journal of Information Systems Education, 21 (3), 313-322. Extraído el 31 de mayo de 2011, de http://proquest.umi.com/pqdlink?RQT $=572 \& V T y p e=P Q D \& V N a m e=P Q D \& V I n s t$ $=$ PROD\&pmid $=50955 \&$ pcid $=55451471 \&$ SrchMode $=3$.

PEKRUN, R.; ELLIOT, A. J. y MAIER, M. A. (2006). Achievement goals and discrete achievement emotions: A theoretical model and prospective test [versión electrónica]. Journal of Educational Psychology, 98 (3), 583-597.

PUZZIFERRO, M. (2008). Online technologies self-efficacy and self-regulated learning as predictors of final grade and satisfaction in college-level online courses [versión electrónica]. The American Journal of Distance Education, 22 (2), 72-89.

RODRÍGUEZ, G. R. y CUEVA, S. P. (2010). OER, estándares y tendencias. RUSC. Revista de Universidad y Sociedad del Conocimiento, 7 (1), 1-8. Extraído el 7 de 
febrero de 2012, de http://rusc.uoc.edu/ojs/index.php/rusc/article/view/v7n1_ cueva_rodriguez.

ROGERSON-REVELL, P. (2007, febrero). Directions in e-learning tools and technologies and their relevance to online distance language education. Open Learning, 22 (1), 57-74. Extraído el 1 de junio de 2011, de http://ehis.ebscohost.com.libezproxy.open. ac.uk/eds/pdfviewer/pdfviewer?vid=11\&hid=20\&sid=9a7e3fc4-5ff1-4909-8022 -510ce92d41d4\%40sessionmgr10 (Acceso restringido Open University).

ROSIE, A. (2000). Online pedagogies and the promotion of "deep learning" [version electronica]. Information Services \& Use, 20 (2/3), 109-116.

SANTOVEÑA, S. M. (2010). Cuestionario de evaluación de la calidad de los cursos virtuales de la UNED. RED. Revista de Educación a Distancia, 25, 1-22. Extraído el 7 de febrero de 2012, de http://www.um.es/ead/red/25/santovena.pdf.

SANTOVEÑA, S. M. (2007). Análisis de cursos de Educación Social en entornos virtuales de aprendizaje y su influencia en la calidad. Enseñanza. Anuario interuniversitario de didáctica, 25, 77-90.

SCLATER, N. (2008). Large-Scale Open Source E-learning Systems at the Open University Uk. Educase. Center for Applied Research [versión electrónica]. Research Bulletin, 12, 1-13.

SMITH, P. A. y HOY, W. K. (2007). Academic optimism and student achievement in urban elementary schools [versión electrónica]. Journal of Educational Administration, 45(5), 556-568. Extraído el 1 de junio de 2011, de http://www.emeraldinsight.com.libezproxy.open.ac.uk/journals.htm?issn=0957-8234\&volume=45\&issue= 5\&articleid=1621690\&show=html\&PHPSESSID=bku75le50h176d751 rkg85go51 (Acceso restringido Open University).

UNIVERSIDAD DE OVIEDO (2009). Catálogo de la Universidad de Oviedo. Extraído el 10 de mayo de 2011, de http://issuu.com/universidadoviedo/docs/catalo goviceinter?viewMode=magazine $\&$ mode $=$ embed.

VICERRECTORADO DE INFORMÁTICA Y COMUNICACIONES (s.f.). Campus virtual. Extraído el 12 de mayo de 2011, de https://www.innova.uniovi.es/innova/campus virtual/campusvirtual.php.

WESTBURG, N. G. y MARTIN, D. (2003). The Relationship between hope, a parent's hope, and student-directed, goal-oriented academic instruction [versión electrónica]. Journal of Humanistic Counseling Education, Education and Development, 42, 152-164. 